\title{
Resolution of conflicting views on thermodynamics of glass transition: A unified model
}

\author{
K T JACOB*, SAGAR PRABHUDEV and R M MALLYA \\ Department of Materials Engineering, Indian Institute of Science, Bangalore 560 012, India
}

MS received 17 May 2010

\begin{abstract}
Classical description of thermodynamic properties during glass transition has been questioned by the entropy-loss model. The uncompensated loss of entropy at the glass transition temperature and zero residual entropy is at the heart of the controversy. Both the models are critically reviewed. A unified model is presented which incorporates features of both entropy loss and residual entropy. It implies two different types of contributions to the entropy of the supercooled liquid, one of which vanishes at the transition and the other which contributes to residual entropy. Entropy gain during spontaneous relaxation of glass, and the nature of heat capacity 'hysteresis' during cooling and heating through the glass transition range support the proposed model. Experiments are outlined for differentiating between the models.
\end{abstract}

Keywords. Glass transition; thermodynamics; classical view; entropy-loss; residual entropy; unified model.

\section{Introduction}

Silica-based optical fibre with impurity levels at parts per billion range used in communication technology is the most visible and critical application of glass in modern times (Papagiakoumou et al 2007). Window glass plays an important role in architectural innovation and optical glasses are used in lenses for microscopes, cameras and telescopes. Carbon-based polymer glasses such as nylon and polyvinyl chloride (PVC) are used to make containers or wraps used in our daily life. Metallic glasses find applications as magnetic materials. Chalcogenide glasses are used in CD-RW computer disks. Switching between glassy and crystalline states in phase-change thin films is exploited for data storage. Phase-change materials (PCMs) can also be used in unified computer memory (PC-RAM) and new types of logic circuit cognitive computing (Raoux et al 2008). Amorphous semiconductors are widely used in photovoltaic cells (Goetzberger et al 2003). Carbohydrate glasses are linked to survival mechanism in living systems under extremes of cold or dehydration. There are applications in food storage and in medicine. Because of the pivotal role of glass in modern technology, it is useful to have clear ideas on the nature of transition from the liquid to glass.

Glass is an amorphous (non-crystalline) solid that can be produced by rapidly cooling a liquid below its glass transition temperature. Glass can also be described as a material, with a solid-like rigidity and liquid-like disorder. The glass can be alternately viewed as a supercooled

*Author for correspondence (katob@materials.iisc.ernet.in) liquid, which is not in internal equilibrium, with a viscosity exceeding $10^{12} \mathrm{~kg} \mathrm{~m}^{-1} \mathrm{~s}^{-1}$.

When a liquid is cooled, it ordinarily solidifies into a crystalline state at its normal freezing point. However, some substances can be supercooled by suppressing spontaneous crystallization. On further cooling such a supercooled liquid undergoes an abrupt change in its specific heat, thermal expansion and compressibility over a narrow range of temperature, well below the freezing point. This 'transition', however, apparently involves no change in enthalpy and specific volume. The temperature at which the transition occurs is called the glass transition temperature $\left(T_{\mathrm{g}}\right)$ and the material that is formed from the supercooled liquid below $T_{\mathrm{g}}$ is called a glass. The transition is not sharp, but occurs over a range of temperature (figure 1). The glass transition temperature depends not only on cooling rate, pressure and composition but also on the method used for determining it. Faster the cooling rate, higher the $T_{\mathrm{g}}$. The glass transition is an irreversible and driven (non-spontaneous) process. The experimentally determined specific heat of glass is very close to that of the crystalline state. The glass can then be viewed as a liquid with certain degrees of freedom frozen-in, which no longer contribute to the specific heat.

Glass transition was thought to be well understood till recently. According to the conventional view the configurational entropy of the supercooled liquid is frozen below $T_{\mathrm{g}}$ all the way to absolute zero. Residual entropy at $0 \mathrm{~K}$ is the characteristic hallmark of the conventional view. The classical understanding on the thermodynamics of glass transition has been challenged by Palmer (1982), Balmakov (1996), Kivelson and Reiss (1999) and Gupta and Mauro (2007). According to these authors, there is an 
entropy loss associated with liquid to glass transition and configurational entropy of glass vanishes at absolute zero. In this article both views are critically examined, experimental methods for testing the two models are discussed, and a reconciliation of the two opposing viewpoints is proposed.

\section{Classical view on glass transition}

At the glass transition temperature, the specific heat, thermal expansivity and compressibility of liquid experience an abrupt drop. But the thermodynamic properties like enthalpy, entropy and the specific volume of the glass so formed apparently remain continuous with that of the liquid. The entropy of glass, $S(g)$, is equal to the entropy of liquid, $S(l)$ at $T_{\mathrm{g}}$.

$$
S(l)=S(g) \text { at } T_{\mathrm{g}} .
$$

On cooling below $T_{\mathrm{g}}$, the entropy of glass decreases and possesses a residual entropy at $0 \mathrm{~K}$ that corresponds approximately to the configurational entropy of glass that was frozen-in at $T_{\mathrm{g}}$. The persistence of residual entropy is not a contradiction to the third law of thermodynamics because the glass represents an excited state, which is not in internal equilibrium.

It is useful to examine the behaviour of excess enthalpy of the glass over that of the crystal, $H_{\text {exc }}(g)=H(g)-H(c)$, as a function of temperature. Here $H(g)$ and $H(c)$ are the enthalpies of the glass and crystal, respectively. Since the measured heat capacity of the glass is only marginally higher than that of the crystalline solid, excess enthalpy of glass given by

$$
H_{\mathrm{exc}}(g)=\int\left(C_{\mathrm{p}}(g)-C_{\mathrm{p}}(c)\right) \mathrm{d} T,
$$

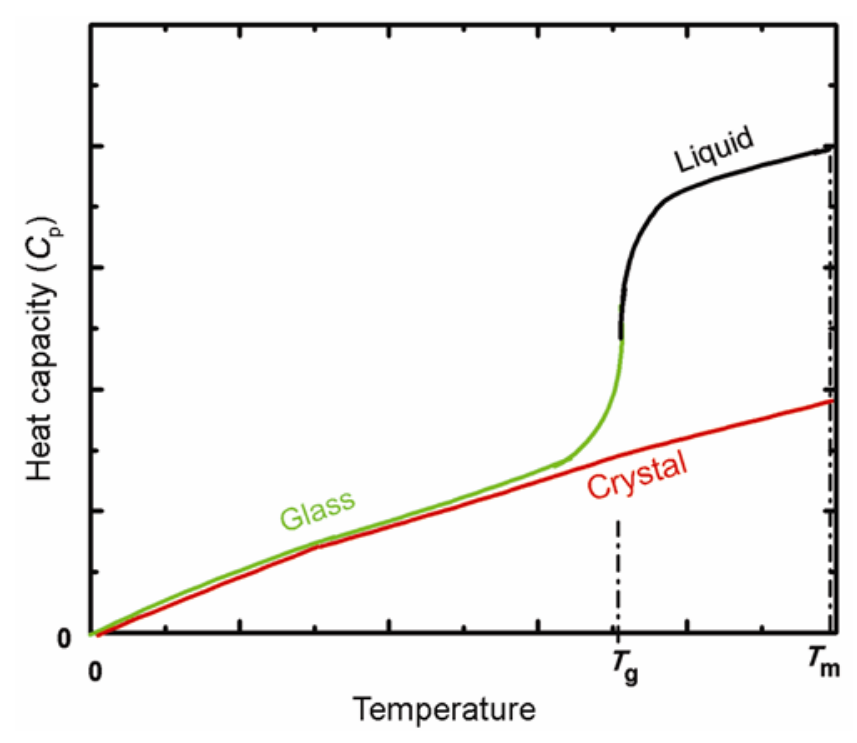

Figure 1. Variation of heat capacities of supercooled liquid, glass and crystalline solid with temperature.
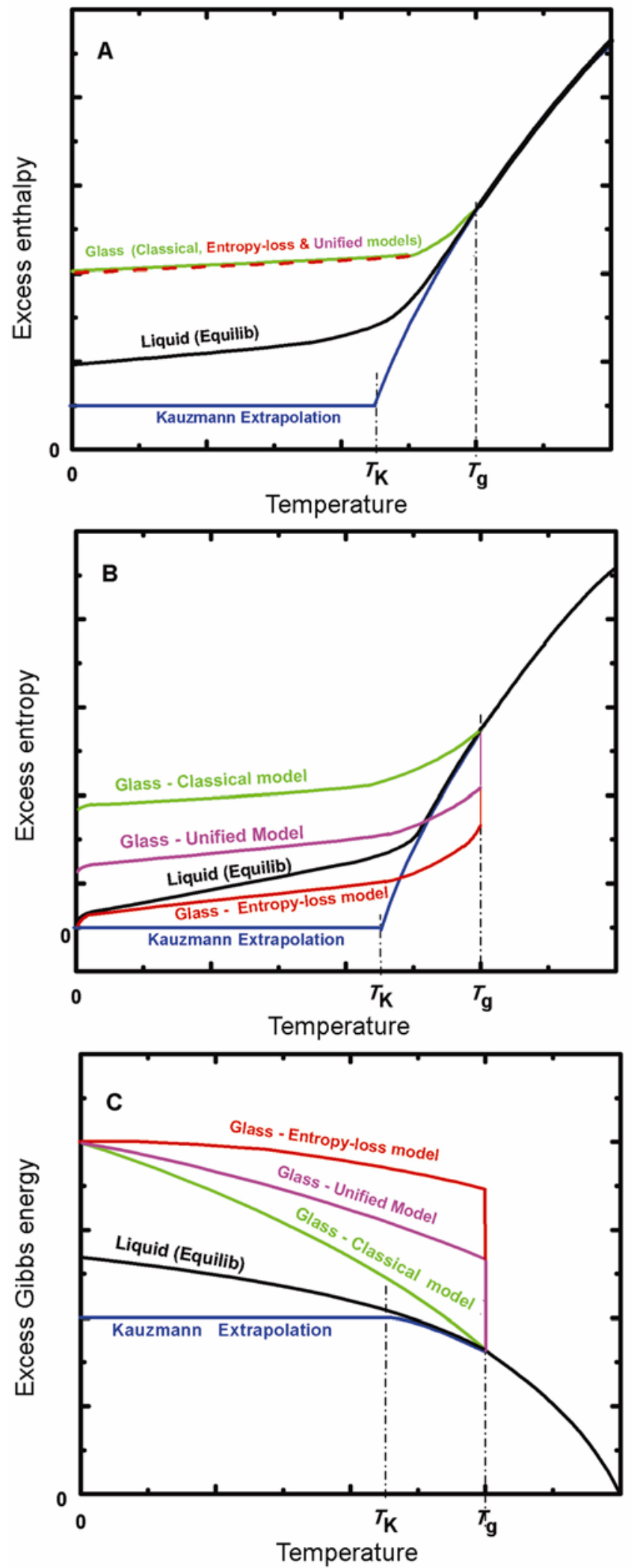

Figure 2. Schematic variation of excess enthalpy (A), excess entropy (B) and excess Gibbs energy (C) of the supercooled liquid and glass relative to the crystalline solid with temperature according to the classical model, the entropy-loss model, the new unified model and the Kauzmann scheme. 
exhibits only mild temperature dependence as shown schematically in figure $2 \mathrm{~A}$. The excess entropy of a glass over that of its crystalline solid, $S_{\text {exc }}(g)=S(g)-S(c)$, varies with temperature as shown in figure $2 \mathrm{~B}$. The excess entropy of glass is defined by the integral,

$$
S_{\mathrm{exc}}(g)=\int \frac{C_{\mathrm{p}}(g)-C_{\mathrm{p}}(c)}{T} \mathrm{~d} T .
$$

Combining excess enthalpy and entropy,

$$
G_{\text {exc }}(g)=H_{\text {exc }}(g)-T S_{\text {exc }}(g) \text {, }
$$

excess Gibbs energy of the glass, $G_{\text {exc }}(g)=G(g)-G(c)$, is obtained as a function of temperature as shown schematically in figure 2C. Quantitative curves for triphenylethene in liquid and glassy states have been presented by Johari $(2000,2010)$.

\section{Thermodynamic properties of equilibrium liquid}

It is useful to keep in view properties of the supercooled liquid in internal equilibrium as a function of temperature for benchmarking the properties of the corresponding glass. Johari (2000) has proposed a method to find the paths of enthalpy and entropy of an internally equilibrated liquid from $T>T_{\mathrm{g}}$ to $0 \mathrm{~K}$ within the constraint of third law of thermodynamics. The most realistic path would be one along which the heat capacity of equilibrium liquid, $C_{\mathrm{p}}(l)$ decreases continuously towards zero at $0 \mathrm{~K}$. Thus, excess enthalpy of the equilibrium liquid over its crystalline solid, $H_{\text {exc }}(l)=H(l)-H(c)$ would have a non-zero value at $0 \mathrm{~K}$, given by

$$
H_{\mathrm{exc}, 0 \mathrm{~K}}(l)=\Delta H_{\mathrm{m}}-\int_{0}^{T_{\mathrm{m}}}\left(C_{\mathrm{p}}(l)-C_{\mathrm{p}}(c)\right) \mathrm{d} T .
$$

The excess entropy of the equilibrium liquid over its crystalline solid, $S_{\text {exc }}(l)=S(l)-S(c)$ becomes zero at $0 \mathrm{~K}$ in accordance with third law. Therefore,

$$
S_{\mathrm{exc}, 0 K}(l)=\Delta S_{\mathrm{m}}-\int_{0}^{T_{\mathrm{m}}} \frac{C_{\mathrm{p}}(l)-C_{\mathrm{p}}(c)}{T} \mathrm{~d} T=0 .
$$

The path along which $C_{\mathrm{p}}(l)$ of the equilibrium liquid would change on cooling below $T_{\mathrm{g}}$ is obtained by interpolating from the known $C_{\mathrm{p}}(l)$ value at $T>T_{\mathrm{g}}$ to $C_{\mathrm{p}}(l)=0$ at $0 \mathrm{~K}$. Since heat capacity of the supercooled liquid below $T_{\mathrm{g}}$ cannot be obtained by experiment or theory, it has to be estimated by an empirical procedure to satisfy (6) and the $T^{3}$ relation at low temperature. The estimated heat capacity profile is not unique.

Excess enthalpy of the equilibrium liquid at $0 \mathrm{~K}$, $H_{\text {exc, } 0 \mathrm{~K}}(l)$, is determined from estimated $C_{\mathrm{p}}(l)$ of supercooled liquid using (5). $H_{\text {exc }}(l)$ can be determined as a function of temperature by evaluating the appropriate area under the plot of $C_{\mathrm{p}}(l)-C_{\mathrm{p}}(c)$ vs temperature. Simi- larly, $S_{\text {exc }}(l)$ is determined as a function of temperature by integration of the plot of $\left(C_{\mathrm{p}}(l)-C_{\mathrm{p}}(c)\right) / T$ vs $T$. Excess Gibbs energy of the equilibrium liquid, $G_{\text {exc }}(l)=G(l)-$ $G(c)$, is obtained by combining its excess enthalpy and excess entropy, $G_{\text {exc }}(l)=H_{\text {exc }}(l)-T S_{\text {exc }}(l)$. Variations of excess enthalpy, excess entropy and excess Gibbs energy of the equilibrium liquid with temperature are shown schematically in figures $2 \mathrm{~A}, 2 \mathrm{~B}$ and $2 \mathrm{C}$, respectively.

\section{Kauzmann paradox}

Because of the translational freedom available in the liquid state, the heat capacity of a liquid, $C_{\mathrm{p}}(l)$ is greater than that of its crystalline solid, $C_{\mathrm{p}}(l)>C_{\mathrm{p}}(c)$. When a liquid is cooled below its freezing temperature, $T_{\mathrm{m}}$, to a temperature $T$, the loss in entropy is given by

$$
\Delta S(l)=\int_{T_{\mathrm{m}}}^{T} \frac{C_{\mathrm{p}}(l)}{T} \mathrm{~d} T .
$$

The crystalline solid also loses entropy on cooling:

$$
\Delta S(c)=\int_{T_{\mathrm{m}}}^{T} \frac{C_{\mathrm{p}}(c)}{T} \mathrm{~d} T .
$$

At the freezing temperature $T_{\mathrm{m}}$, the entropy of liquid exceeds that of its crystalline solid by $\Delta S_{\mathrm{m}}$, the entropy of fusion. Since $C_{\mathrm{p}}(l)>C_{\mathrm{p}}(c)$, the entropy lost by the liquid on cooling would be greater than that of the crystalline solid. At $T=T_{\mathrm{K}}$, entropy of the liquid becomes equal to that of the solid. At temperatures below $T_{\mathrm{K}}$, the entropy of liquid becomes lower than that of the solid, which is physically inconceivable except in some rare situations. This paradox was first recognized by Kauzmann (1948). Thus, $T_{\mathrm{K}}$ (Kauzmann temperature) is often considered to be the low temperature limit for the stability of the supercooled liquid. $T_{\mathrm{K}}$ is mathematically defined by

$$
\int_{T_{\mathrm{m}}}^{T_{\mathrm{K}}} \frac{C_{\mathrm{p}}(c)-C_{\mathrm{p}}(l)}{T} \mathrm{~d} T=\Delta S_{\mathrm{m}}
$$

Supercooled liquid should transform into a glass at or above this temperature to prevent an entropy catastrophe, i.e. entropy of the liquid being less than that of the solid. The liquid would lose entropy equivalent to its entropy of fusion on supercooling from $T_{\mathrm{m}}$ to $T_{\mathrm{K}}$. In the Kauzmann model, the excess entropy becomes zero at temperatures lower than $T_{\mathrm{K}}$ as shown in figure $2 \mathrm{~B}$. The excess enthalpy of the liquid, $H_{\mathrm{exc}}(l)$, would decrease till $T_{\mathrm{K}}$ is reached in the Kauzmann scheme, and then remains approximately constant as shown in figure $2 \mathrm{~A}$. The excess Gibbs energy of the liquid/glass in the Kauzmann scheme is obtained by combining the corresponding excess enthalpy and 
excess entropy and is displayed in figure 2C. Kauzmann temperature is a limiting concept and cannot be reached in practice (Kauzmann 1948).

\section{Entropy-loss model}

The classical understanding of the glass transition has been questioned recently (Palmer 1982; Balmakov 1996; Kivelson and Reiss 1999; Gupta and Mauro 2007; Reiss 2009). According to these authors, the configurational entropy goes to zero at glass transition temperature as the molecular motion is frozen into a single microstate and cannot visit the alternative degenerate states during the time of measurement. Thus $S(l) \neq S(g)$ at $T_{\mathrm{g}}$. This contradicts the classical view discussed above (1), according to which the entropy of the glass should be equal to that of the supercooled liquid at $T_{\mathrm{g}}$.

The loss of entropy at $T_{\mathrm{g}}$ is approximately equal in magnitude to the residual entropy at $0 \mathrm{~K}$ in the classical thermodynamic understanding of glass transition. According to Reiss (2009) there is no residual entropy at $0 \mathrm{~K}$. Residual entropy is an artefact resulting from measurements along partially irreversible paths.

The measured heat capacity during glass formation is known to depend on the time taken for measurement. According to Reiss (2009), the heat capacity during glass transition is not a state property as it is a function of the cooling rate. Heat capacity measured during cooling and heating experiments are different in the temperature range around the glass transition. During heating a spike (overshoot) in heat capacity (Angell and Torrell 1983) is observed as shown schematically in figure 3 . Therefore, integral of the measured $C_{\mathrm{p}}$ cannot give a true measure of entropy. Reiss argues that residual entropy of the glass derived from measured heat capacities is an apparent quantity.

The variation of excess entropy of glass relative to the crystal as a function of temperature according to entropyloss model is shown in figure $2 \mathrm{~B}$. Both classical and entropy-loss models concur regarding the variation of excess enthalpy with temperature. Experimentally no significant heat effects have been detected during glass transition in support of the schematic variation of excess enthalpy with temperature shown in figure 2A. Discontinuity in entropy accompanied by continuity in enthalpy at $T_{\mathrm{g}}$ may appear to violate conventional understanding of phase transitions. However, glass transition is an irreversible process and not a true thermodynamic phase transition. It has kinetic rather than thermodynamic origin. The glassy state is always thermodynamically unstable, but kinetically stable. The glass and corresponding liquid phase cannot be connected by any path in a time-independent parameter space.

The excess Gibbs energy according to the entropy-loss model is obtained by combining the excess enthalpy and excess entropy. Its variation with temperature is shown in figure $2 \mathrm{C}$. Entropy-loss model suggests a sharp increase in excess Gibbs energy at $T_{\mathrm{g}}$ on cooling.

The physics of entropy-loss view rests on the issue of ergodicity. Ergodic state corresponds to an equilibrated disordered structure in which diffusion occurs in the experimental time frame and whose properties do not change with time. Glass transition is seen as an "ergodicity breaking' process in which system goes from an ergodic (equilibrium liquid) to a non-ergodic state (glass). Interpretation of Boltzmann's entropy is at the centre of the controversy. In statistical thermodynamics, $S_{\text {conf }}=k_{\mathrm{B}} \ln \Omega$, where $k_{\mathrm{B}}$ is the Boltzmann constant and $\Omega$ the number of configurations of equal energy. Does $\Omega$ refer to microstates actually accessed by the system during the time of observation or is it determined by all accessible states, irrespective of whether they are actually sampled? The controversy revolves around 'accessible' (available) versus 'accessed' microstates.

\section{Discussion on entropy-loss model}

Entropy-loss model has been recently critiqued by Goldstein (2008). He argues that the spontaneous decrease in entropy at glass transition implies directly the possibility of an uncompensated conversion of heat to work in violation of the second law of thermodynamics. He notes that the number of microstates visited in the course of a measurement does not determine entropy. This number is always an inconceivably small fraction of accessible microstates. Entropy is determined by the microstates that are equally accessible. In a rebuttal of the critique of Goldstein, Gupta and Mauro (2008) state that there is no violation of second law because glass transition is not a spontaneous process, since it is the inverse of glass relaxation which is spontaneous. Reiss (2009) has suggested that the concept of residual entropy violates the fundamental principle of causality.

While conceding that heat capacity in the region of glass transition is not a true state function, Goldstein (2008) maintains that experiments conducted at different cooling rates can set upper and lower bounds for heat capacity variation with temperature. Integrating along the upper and lower bounds can provide an estimate of the error in computing residual entropy at $0 \mathrm{~K}$. Because of the relatively small range of temperature for the glass transition, the extreme $C_{\mathrm{p}}$ profiles mainly affect the magnitude of estimated residual entropy, but do not negate it. The $C_{\mathrm{p}}$ variations only introduce uncertainty in the estimated residual entropy (Goldstein 2008).

If there is entropy loss at the glass transition without any change in enthalpy, it cannot be directly detected or its magnitude assessed by calorimetry. However, the magnitude and temperature dependence of the heat capacity before and after the transition can give an indication 
of the underlying mechanisms. The sharp drop in heat capacity at the glass transition during cooling suggests loss of certain modes of energy absorption probably involving translational motion of atoms or molecules. The switching off of translational freedom must be associated with an entropy loss. A sharp increase in heat capacity is observed on heating the glass (figure 3) (Angell and Torrell 1983). The higher heat capacity on heating and the associated spike are indicative of the onset of new contributions to heat capacity - gain of an additional degree of freedom accompanying glass relaxation.

\section{Reconciliation of classical and entropy-loss views of glass transition: a unified model}

Contrary to the opinion expressed by Johari (2010), the classical and entropy-loss models of glass transition are not necessarily mutually exclusive. There may be elements of truth in each of these models. A critical assessment of the arguments for and against the entropy-loss model in the light of available experimental data appear to suggest the possibility of a partial entropy loss at glass transition as well as significant residual entropy at $0 \mathrm{~K}$. Presence of both components is supported by the observed 'hysteresis' in heat capacity near the glass transition (Reiss 2009) and noticeable entropy gain during relaxation of the glass. Calorimetry cannot reveal an entropy change without any heat effect at the glass transition. However, available calorimetric heat capacity data, even after correcting for the effect of irreversibility during glass transition, still suggests some residual entropy at $0 \mathrm{~K}$ (Hikima et al 1998). If all the excess entropy of supercooled liquid at the glass transition temperature were frozen as residual entropy, then one should not see significant entropy change accompanying the relaxation process. There is a

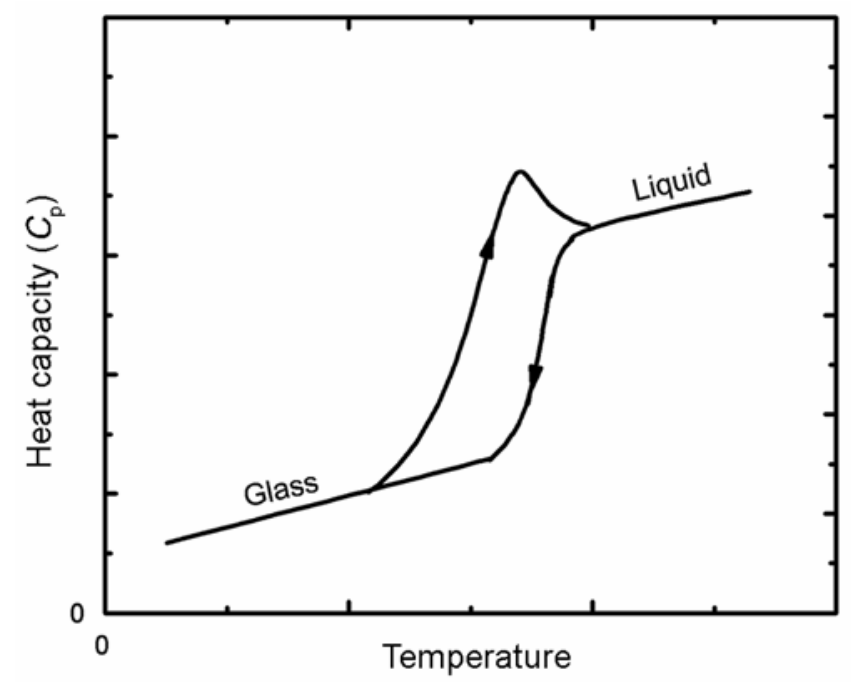

Figure 3. Heat capacity variations in glass transition region during heating and cooling (Angell and Torrell 1983) link between entropy loss during glass transition and entropy gain during relaxation as suggested by Gupta and Mauro (2008). Glass transition is the inverse of glass relaxation. Glass relaxation being a spontaneous process is associated with an increase in entropy. The hysteresis in heat capacity during cooling and heating (figure 3) is indicative of partial entropy loss during glass transition and entropy gain during relaxation. On the one hand, some degrees of freedom, notably involving translation and in some cases rotation, is lost at the glass transition with consequent loss of entropy. On the other hand, other types of defects, akin to those in disordered solids, persist at low temperatures contributing to residual entropy. Part of the disorder in the glass is manifested in structural studies of glasses. The frequently observed deformed pattern in pair distribution function and structure factor of glasses can be interpreted as a slight spacial inhomogeneity in the average atomic distribution (Waseda 1980). Recognition of the degrees of freedom and disorder in the supercooled liquid is important for understanding the simultaneous existence of entropy-loss and residual entropy. The excess entropy of the liquid can be visualized as having two components. For monoatomic fluids or those made up of non-polar spherical molecules, a significant part of the excess entropy is communal entropy, which is essentially translational entropy. Loss of translational freedom arises from changes in interatomic or intermolecular forces on cooling. The loss is not entirely caused by kinetic factors. The second component of the excess entropy of the liquid is associated with structural defects such as vacancies and lack of longrange order. Loss of translational freedom affects entropy in a manner different from the freezing-in of defects on rapidly cooling the glass. The frozen defect states contribute to residual entropy. The exact partitioning of the excess entropy of the supercooled liquid at the glass transition temperature between a loss component at the transition temperature and residual entropy at $0 \mathrm{~K}$ needs to be evaluated carefully by experiment. The partition ratio may be expected to vary somewhat for different types of glasses.

There are two phenomena that occur simultaneously during the transformation of a liquid into a crystalline solid: the atoms loose their translational freedom and they acquire long range order. During the transition of the supercooled liquid into glass, the atoms loose their translational freedom but retain the disorder of the liquid state. The glass has only short-range order and lacks long-range periodicity in the arrangement of atoms. The partial loss of entropy at the glass transition is linked to the loss of translational freedom and the residual entropy of the glass is caused by the retained configurational or positional disorder.

\section{Experimental verification}

Ultimate validation of the three different thermodynamic models of glass transition discussed above must rest on 
sound experimental evidence. While there is some scanty information in the literature on vapour pressure and solubility of glass in comparison to that of the corresponding crystal, the experiments have not been specifically performed to test the models and several critical issues are not addressed. In the discussion that follows the methods available for testing, system and characterization requirements are outlined.

Since there is no difference in the variation of excess enthalpy of the glass in the three models as shown in figure $2 \mathrm{~A}$, heat content measurements cannot provide differentiation. The differences between the models show up only in the excess entropy and excess Gibbs energy as shown in figures $2 \mathrm{~B}$ and $2 \mathrm{C}$. Since entropy cannot be directly measured, the only avenue available for the experimental verification is the measurement of Gibbs energy of the glass below $T_{\mathrm{g}}$. The excess Gibbs energy can be most conveniently measured using suitably designed electrochemical cells. The glass phase can be used as a measuring electrode and the crystalline phase as the reference electrode of the cell. The electrochemical method is most suited for unary or binary systems. This method becomes more complicated for multicomponent glass systems since the electrochemical potential is usually generated by the exchange of only one species at the electrode-electrolyte interface. Thus only partial Gibbs free energy of one component of the glass is directly measurable and there is no generally applicable method for obtaining the integral Gibbs energy of the glass. In some specific cases it may be possible to define partial free energies of other components by introducing suitable auxiliary phases at the electrode.

Relative solubility of the glass and crystalline phases in suitable solvents can also provide a measure of excess Gibbs energy under suitable conditions. During Gibbs energy measurements, the glass surface is always in equilibrium with another phase, be it the electrolyte, solvent or vapour. The equilibrium exchange of material between the glass and the second phase in equilibrium can lead to reconstitution of the glass surface. Hence it would be necessary to conduct the measurement as rapidly as possible and to ensure that surface reconstitution has not occurred during measurement using sensitive surface analytical techniques. Most glassy materials, especially metal and oxide glasses, may not have significant vapour pressure below the glass transition temperature to exploit it for measurement of the Gibbs energy. If the glassy phase has sufficient vapour pressure and vaporization is congruent, Langmuir free evaporation in high vacuum may be more appropriate than static or dynamic Knudsen (equilibrium) effusion methods. Since vapour species emanating from the surface of the solid are continuously removed by vacuum, surface reconstruction will be minimal in the Langmuir evaporation method. However, to relate mass loss to vapour pressure, evaporation coefficient should be known. If the evaporation coefficient is the same for the different states (glass and solid) of the same material, mass loss will be proportional to vapour pressure and one can map the relative variation of Gibbs energy with temperature in the vicinity of glass transition. The use of solubility and vapour pressure measurements to differentiate between the classical and entropy-loss models has been discussed by Goldstein (2008) for simple systems. In multicomponent glasses preferential vaporization or dissolution of a component of the glass can limit the applicability of these methods. Congruent vaporization or dissolution has to be experimentally established for the valid use of these methods.

\section{Conclusions}

Classical and entropy-loss views of the thermodynamics of glass transition have been critically examined in the light of available experimental data. The points of agreement and conflict are identified. In the classical view there is no entropy change at the glass transition temperature and there is residual entropy at $0 \mathrm{~K}$. In the entropyloss model there is entropy loss at the glass transition temperature and no residual entropy at $0 \mathrm{~K}$. Contrary to the suggestion of Johari (2010), features of the two models are not necessarily mutually exclusive. A unified model is presented in which partial loss of the excess entropy of the supercooled liquid-mainly related to translational freedom-occurs at the transition temperature, and the remainder associated with disorder is present as residual entropy. The proposed model is based on essentially two different types of contributions to the excess entropy of the supercooled liquid, one of which vanishes at the glass transition temperature and the other persists to low temperatures resulting in residual entropy. This interpretation is consistent with the experimental information on heat capacity hysteresis in the temperature range of glass transition and data on entropy gain during relaxation of the glass.

\section{Acknowledgements}

One of the authors (KTJ) acknowledges the award of a distinguished professorship by the Indian National Academy of Engineering which made this work possible.

\section{References}

Angell C A and Torell L M 1983 J. Chem. Phys. 78937

Balmakov M D 1996 J. Glass Phys. Chem. 22344

Goetzberger A, Hebling C and Schock H W 2003 J. Mater. Sci. Eng. 401

Goldstein M 2008 J. Chem. Phys. 128154510

Gupta P K and Mauro J C 2007 J. Chem. Phys. 126224504

Gupta P K and Mauro J C 2008 J. Chem. Phys. 129067101

Hikima T, Okamoto N, Hanaya M and Oguni M 1998 J. Chem. Thermodynamics 30509 
Johari G P 2000 J. Chem. Phys. 113751

Johari G P 2010 Thermochim. Acta 500

Kauzmann W 1948 Chem. Rev. 43219

Kivelson D and Reiss H 1999 J. Phys. Chem. B103 8337

Palmer R G 1982 J. Adv. Phys. 3169

Papagiakoumou E, Papadopoulos D N and Serafetinides A A 2007 J. Opt. Commun. 27680
Raoux S, Shelby R M, Sweet J J, Munoz B, Salinga M, Chen Y, Lai E K and Lee MH 2008 J. Microelectron. Eng. 85 2330

Reiss H 2009 J. Non-Cryst. Solids 355617

Speedy R J 2003 Biophys. Chem. 105411

Waseda Y 1980 The structure of non-crystalline materials (New York: McGraw-Hill International Book Co.) 